\title{
USO DE DEFENSIVOS AGRÍCOLAS, LIMITES MÁXIMOS DE RESÍDUOS E IMPACTO NO COMÉRCIO INTERNACIONAL: ESTUDO DE CASO $^{1}$
}

\author{
Ricardo Kropf Santos Fermam ${ }^{2}$ \\ Adelaide Maria de Souza Antunes ${ }^{3}$
}

Resumo: Os defensivos agrícolas desempenham importante papel na agricultura, já que previnem perdas devido à ação de pragas que destroem as plantações e causam inúmeros prejuízos aos produtores, bem como nos países exportadores de produtos agrícolas. Contudo, os defensivos deixam resíduos onde quer que sejam empregados. Um dos parâmetros utilizados na quantificação desses resíduos é conhecido como Limite Máximo de Resíduo (LMR). Este parâmetro é o mais importante não só para a saúde pública, mas também para o comércio internacional, visto que ocasiona diversos impactos que podem representar, em alguns casos, obstáculos ao comércio entre os países, especialmente quando os valores estabelecidos por um país diferem dos estabelecidos por organizações internacionais. O presente artigo mostra como esse parâmetro impacta o comércio de produtos agrícolas de países em desenvolvimento, baseado num estudo de caso.

Palavras-chave: Defensivos, resíduo, LMR, comércio internacional.

\section{Introdução}

Os defensivos agrícolas desempenham importante papel na agricultura, visto que previnem perdas de produtividade devido a ervas daninhas, insetos, fungos, dentre outros. Sem eles, a demanda de alimentos tornaria rapidamente maior do que a oferta, em razão de perdas por pragas. Por isso, são produtos-chave para garantir a competitividade do setor agrícola, num mundo globalizado.

Recebido em: 28/04/09; Aceito em: 09/09/09.

2 Doutorando do Programa de Pós-Graduação em Tecnologia de Processos Químicos e Bioquímicos, Escola de Química, UFRJ.E-mail: rkfermam@inmetro.gov.br.

3 Professora Titular (D.Sc.) do Programa de Pós-Graduação em Tecnologia de Processos Químicos e Bioquímicos, Escola de Química, UFRJ. E-mail: adelaide@eq.ufrj.br. 
A utilização de defensivos agrícolas na agricultura é vasta e extensiva, na grande maioria das culturas. Em geral, o uso de defensivos tem crescido bastante, pois a busca de maiores produtividades é cada vez mais importante para a sobrevivência dos negócios. O controle de pragas, pela aplicação de herbicidas, fungicidas, acaricidas e inseticidas, é um dos maiores desafios dos produtores agrícolas. Se tal controle não for eficiente, poderá haver substancial redução na produtividade das culturas e na rentabilidade da atividade produtiva e aumento nos preços dos produtos agrícolas.

Há que se ponderar, contudo, que os defensivos agrícolas são, talvez, os produtos mais sujeitos a rígidas legislações no mundo. Cada vez mais, os países estabelecem requisitos técnicos, por meio de normas técnicas, regulamentos técnicos e avaliação da conformidade (por exemplo, laboratórios de ensaio) não só para produção, aplicação, exportação e importação dessas substâncias, bem como para as culturas em que tais substâncias são empregadas. Esse fato se deve, principalmente, à alta periculosidade dos defensivos agrícolas para a saúde das pessoas e para o meio ambiente.

Assim, os produtos do agronegócio, sobretudo os mais competitivos, enfrentam forte protecionismo nos mercados externos. Em geral, há dois tipos de medidas protecionistas aos produtos agrícolas: as tarifárias (picos tarifários, escaladas tarifárias, tarifas proibitivas, quotas tarifárias, salvaguardas agrícolas especiais, etc) e as não-tarifárias (barreiras técnicas, fitossanitárias, subsídios, etc).

De maneira geral, os requisitos técnicos adotados para produtos agroalimentares, nos países desenvolvidos, são bem mais altos que os prevalecentes em países em desenvolvimento, envolvendo custos mais elevados. Além disso, os requisitos referentes a aspectos de segurança e qualidade desses produtos têm-se alterado com frequência, devido à maior velocidade com que novas tecnologias são desenvolvidas e adotadas, tanto para obtenção de produtos mais adequados ao consumo, como para o controle da qualidade desses produtos nos vários estágios de produção, processamento e comercialização (OLIVEIRA, 2005). 
Tais requisitos estão relacionados com diversos "elos" da cadeia produtiva do agronegócio, em especial, com os insumos provenientes da indústria química, como os defensivos agrícolas. Limites máximos de resíduos, métodos e processos de produção, tipos de substâncias permitidas ou proibidas, rotulagem, dentre muitas outras exigências técnicas, por parte dos países importadores, acabam produzindo barreiras comerciais não só dessas substâncias, mas também dos produtos do agronegócio, principal usuário dessas substâncias.

\section{O Limite Máximo de Resíduo (LMR)}

Os defensivos deixam resíduos onde quer que sejam empregados, às vezes inalterados (em sua forma química original) e, muitas vezes, sob a forma de produtos degradados, até que ocorra a sua degradação final, cuja duração é variável. No ambiente, alguns mais persistentes podem até passar de uma cultura para um animal, e desse animal para o homem 4 . Por essa razão é que se estabelece em lei a quantidade máxima de resíduos desse defensivo permitida para determinado produto agrícola, valor denominado de tolerância ou limite máximo de resíduos (FERREIRA, 1987).

O estabelecimento e a regulamentação de limites máximos de resíduos (LMR) em alimentos, por agências governamentais de diversos países e por órgãos internacionais ${ }^{5}$, têm se tornado mais frequentes na última década. Esses procedimentos, em geral, visam garantir a segurança do consumidor e a regulamentação do comércio externo (JARDIM e ANDRADE, 2009). Assim, o referido parâmetro é o mais importante não só para a saúde pública, mas principalmente para o comércio internacional.

\footnotetext{
${ }_{4}$ Ressalta-se que a decomposição de alguns ingredientes ativos pode produzir substâncias mais perigosas do que o ingrediente em si, como, por exemplo, no caso do Ethefon, que, em meio alcalino ou neutro, libera gás etileno, que é inflamável.

5 No âmbito internacional, esses limites são estabelecidos pela Comissão do Codex Alimentarius, segundo recomendações do grupo de peritos em resíduos de defensivos das Organizações das Nações Unidas para a Agricultura e Alimentação (FAO), Organização Mundial de Saúde (OMS) e Joint Meeting on Pesticides Residues (JMPR).
} 
O limite máximo de resíduos (LMR) é a quantidade máxima de resíduo ${ }^{6}$ de defensivo, ou afim, oficialmente aceita no alimento, em decorrência da aplicação adequada numa fase específica, desde sua produção até o consumo, expressa em miligramas do defensivo afim ou de seus resíduos por quilo do alimento analisado $(\mathrm{mg} / \mathrm{Kg})$. Por exemplo, LMR da azociclotina, defensivo utilizado no Brasil nas culturas de café, citros, feijão, maçã e tomate, é igual à soma de azociclotina, cihexatina e diciclohexilestanho, expressos em cihexatina. As rotas de degradação da azociclotina são mostradas na Figura 1.

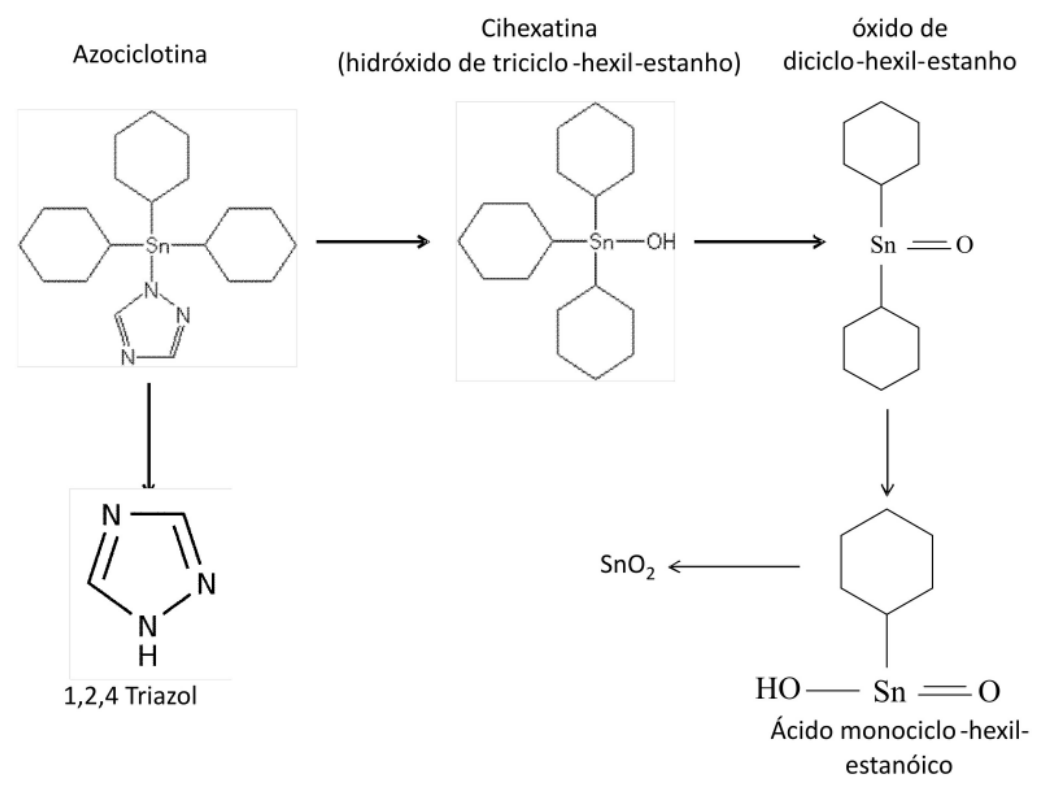

Figura 1 - Rotas de degradação da azociclotina.

6 Resíduo: Substância ou mistura de substâncias remanescente ou existentes em alimentos ou no meio ambiente, decorrente do uso ou da presença de defensivos e afins, inclusive quaisquer derivados específicos, tais como produtos de conversão e de degradação, metabolitos, produtos de reação e impurezas, consideradas toxicológica e ambientalmente importantes. Exemplo: Azociclotina - resíduos: azociclotina, cihexatina e diciclohexilestanho. 
No estudo de resíduos, o ácido monociclo-hexil-estanóico sempre representa menos que $10 \%$ do total de resíduos. Já o 1,2,4 Triazol não possui significância analítica ou encontra-se abaixo do limite de detecção. Portanto, esses compostos não são considerados para efeitos de LMR (IPCS INCHEM, 1979).

Os limites máximos de resíduos referem-se a valores provenientes de experimentos de campo, exigidos para cada cultura alimentar, e levam em consideração a Ingestão Diária Aceitável (IDA), que é a quantidade máxima que, ingerida diariamente durante toda a vida, parece não oferecer risco apreciável à saúde ${ }^{7}$, à luz dos conhecimentos atuais. É expressa em $\mathrm{mg}$ do agrotóxico em $\mathrm{kg}$ de peso corpóreo (mg/kg p.c.).

A IDA, determinada para cada ingrediente ativo de defensivo, é estabelecida com base em estudos sobre as propriedades físico-químicas, metabólicas, farmacológicas e toxicológicas dos defensivos, advindas dos estudos conduzidos com animais de laboratórios e realizados em procedimentos reconhecidos em nível internacional ${ }^{8}$.

Para o correto estabelecimento de LMR de determinado ingrediente ativo em um alimento, é necessário levar em consideração as condições edafoclimáticas ${ }^{9}$, as pragas presentes no ambiente, as indicações de dose e forma de aplicação, bem como o intervalo de segurança proposto. Esse conjunto de fatores, que interferem no resultado do LMR, é denominado de Boas Práticas Agrícolas (BPA). Tais fatores são variáveis

\footnotetext{
Exemplificando: se determinado ingrediente ativo, contido em um defensivo, tiver uma IDA igual a 0,05 mg/ $\mathrm{kg}$, significa que uma pessoa de $60 \mathrm{~kg}$, por exemplo, poderia ingerir uma quantidade máxima de 3,0 mg, diariamente, sem riscos à saúde.

8 Organização Mundial da Saúde (OMS), Programa Internacional de Segurança de Substâncias Químicas (IPCS / OMS), Agência Internacional de Pesquisas Sobre o Câncer (IARC/OMS), Centro Pan-Americano de Ecologia Humana e Saúde (ECO/OPS), Organização das Nações Unidas para a Agricultura e Alimentação (FAO), Registro Internacional de Substâncias Potencialmente Tóxicas do Programa das Nações Unidas para o Meio Ambiente (IRPTC/UNEP), Organização para Cooperação Econômica e Desenvolvimento da Comunidade Econômica Européia (OECD/CEE) e Agência de Proteção Ambiental dos Estados Unidos da América (EPA).

9 A expressão condições edafoclimáticas refere-se às características definidas pelos fatores do meio ambiente, tais como clima, relevo, litologia, temperatura, umidade do ar, radiação, tipo de solo, vento, composição atmosférica e precipitação pluvial. As condições edafoclimáticas são relativas à influência dos solos nos seres vivos, em particular nos organismos do reino vegetal, incluindo o uso da terra pelo homem, a fim de estimular o crescimento das plantas.
} 
e precisam ser levados em consideração no momento da elaboração de critérios para o estabelecimento de limites máximos de resíduos ${ }^{10}$.

Desse modo, por meio do LMR é possível verificar se as recomendações indicadas pelos fabricantes nos rótulos e nas bulas de seus defensivos estão sendo seguidas, assim como prever o consumo de resíduos de defensivos em alimentos, de forma segura à saúde humana, quando da aplicação desse defensivo conforme boas práticas agrícolas.

Durante a avaliação dos estudos toxicológicos, é definido o NOEL (NoObserved-Effect-Level) ou o NOAEL (No-Observed-Adverse-EffectLevel), que correspondem à maior concentração ou quantidade do defensivo, em $\mathrm{mg} / \mathrm{kg}$ p.c., determinada experimentalmente, a qual não causa reações ou reações adversas detectáveis, respectivamente, no organismo exposto.

O NOAEL pode ser diferente em virtude de diferentes interpretações ou julgamentos profissionais e também é dependente do tamanho da população em estudo. Estudos que abrangem grande número de animais aumentam a probabilidade de se definirem doses mais baixas, como valores, de NOAEL. O resultado crítico utilizado na avaliação doseresposta é aquele efeito que apresenta o menor NOAEL (NOVAES, 2000).

A avaliação dose-resposta é o processo de vincular, quantitativamente, a informação da toxicidade (incidência do efeito adverso à saúde na população exposta) e a caracterização da relação entre a dose de toxicante administrada ou recebida. Essa avaliação implica considerar três problemas (FREITAS, 2002):

- Os animais em estudos experimentais, geralmente, estão expostos a doses altas, e os efeitos a doses baixas em humanos devem ser previstos pelas teorias relativas na forma da curva dose-resposta;

${ }^{10}$ Os LMRs são índices que podem ser alterados de acordo com novas evidências científicas que indiquem potencial risco à saúde, novas técnicas laboratoriais e, ou agrícolas e desinteresse das empresas em produzir, podendo ter seus valores alterados ou até mesmo extintos. 
- Os animais e os seres humanos, frequentemente, diferem em suscetibilidade, ao menos em diferença de tamanho e metabolismo (variabilidade interespécies);

- A população humana é muito heterogênea, razão por que alguns indivíduos são mais suscetíveis que a média (variabilidade intraespécie).

Na determinação da IDA ${ }^{11}$ aplica-se um fator de segurança arbitrário ao NOAEL ou NOEL, que leva em consideração o tipo de efeito, a severidade ou a reversibilidade desse efeito, bem como os problemas de variabilidade inter e intraespécies. O fator de segurança ${ }^{12} 100$ é geralmente utilizado, pois comporta uma variação dentro da espécie testada (10), multiplicada por uma variação entre a espécie testada e o homem (10). ]

\section{Requisitos internacionais referentes ao Limite Máximo de Resíduo}

Do ponto de vista das regras do comércio internacional, os limites máximos de resíduos de defensivos agrícolas para diversas culturas são estabelecidos pela Comissão do Codex Alimentarius. A Comissão do Codex Alimentarius é uma organização inter-governamental internacional criada em 1963, com o intuito de oferecer respostas a duas preocupações: proteger a saúde dos consumidores e garantir práticas equitativas no comércio de alimentos ${ }^{13}$.

\footnotetext{
11 Da mesma forma que o LMR, a IDA não é um índice permanente. Pode ser alterado por meio de novas avaliações toxicológicas, desde que haja conhecimento científico que indique que não há segurança no índice anteriormente estabelecido.

12 Admite-se que os humanos sejam 10 vezes mais sensíveis do que os animais usados nos testes e que a variação de sensibilidade na população humana atinja um intervalo de 10 vezes. Em alguns casos, o fator de segurança de 100 é considerado insuficiente, podendo-se aplicar valores mais altos de 200, 500, 1000 e 2000 (Ex: estudos incompletos e inadequados, ou quando efeitos irreversíveis são observados como teratogênese e carcinogênese).

13 Comissão do Codex Alimentarius, Procedural Manual, $8^{a}$ edição (1993): 39. Veja-se em www.codexalimentarius.net.
} 
A Comissão do Codex Alimentarius é uma coleção de códigos de práticas e padrões para alimentos, apresentados de maneira uniforme. Seus objetivos são o estabelecimento de códigos de práticas e padrões para proteger a saúde do consumidor e garantir práticas justas ao comércio de alimentos, além de orientação e estímulo ao estabelecimento de definições e exigências para com alimentos, visando promover sua harmonização e facilitar o comércio internacional.

O Codex é o mais importante dos organismos internacionais que desenvolvem padrões de inocuidade e qualidade nos alimentos. Atualmente, o Codex conta com mais de 165 países-membros, que enviam delegações aos subcomitês do Codex, cuja principal tarefa é a produção normativa. O Codex conta com dois tipos principais de comitês: os comitês de produtos (commodities) ou comitês verticais, que fixam padrões para cada um dos produtos básicos, e os comitês de questões gerais ou horizontais, que tratam, entre outras coisas, de questões como os remédios veterinários, aditivos químicos e contaminantes e resíduos de defensivos (POST, 2003).

É oportuno ressaltar que o Comitê do Codex Alimentarius sobre Resíduos de Defensivos (Codex Committee on Pesticide Residues - CCPR), em sua $39^{\text {a }}$. Sessão, realizada em Beijing/China, 7 - 12 de maio de 2007, discutiu a questão da necessidade de enforcement dos LMRs, em nível nacional (especialmente para commodities importadas), determinados por aquela organização (CX/PR 07/39/10).

Durante as discussões, foram identificados vários problemas que afetam as exportações e importações, tais como LMRs nacionais menores que os estabelecidos pelo Codex, produtos que estavam em conformidade com os LMRs estabelecidos pelo país importador, mas que eram reexportados para outro país com LMRs diferentes, e limites impostos por compradores ou organismos de acreditação.

Além disso, evidenciou-se que os países exportadores, particularmente os que estão em desenvolvimento, deparam-se com diferentes valores de LMRs estabelecidos pelos países importadores, para o mesmo 
composto e mesma commodity. Em alguns casos, quando o país exportador detecta valores que não estejam em conformidade com o LMR do país importador, mas que estão de acordo com o Codex, a commodity não é exportada, porém é vendida no mercado local e consumida domesticamente.

O CCPR recomendou que, a fim de avaliar a magnitude e a seriedade desse problema, os países-membros do Codex compilem toda informação disponível, comparando os LMRs nacionais com os estabelecidos pelo Codex, dado que há indícios de desvios em vários países. Deve também ser considerado o fato de que os LMRs, do Codex, são estabelecidos com base numa avaliação de risco $^{14}$, em escala mundial, e são reconhecidos como benchmark internacional pela OMC, no contexto do SPS/OMC. Desse modo, a prática de impor limites, sem justificativa científica, é considerada barreira ao comércio.

Os LMRs são medidas sanitárias que podem afetar o acesso a mercados; desse modo, devem ser considerados no âmbito do acordo SPS/OMC. Assim, devem ser harmonizados em normas internacionais, para evitar que configurem restrições injustificadas ao comércio internacional. É também importante ressaltar que em muitos dos ingredientes ativos usados por países exportadores de alimentos, em relação aos produtos aplicados, não há LMRs estabelecidos pelo Codex, como no caso da Argentina ${ }^{15}$ (G/SPS/W/211).

Países exportadores adotam, frequentemente, os LMRs para defensivos estabelecidos pelos mercados importadores ou removem os registros dos defensivos para assegurar que seus produtos tenham acesso a mercados externos. Isso resulta em altos custos de produção, com sérias consequências na competitividade das exportações dos países em desenvolvimento que não subsidiam as exportações de produto.

\footnotetext{
$\overline{14}$ Processo científico consiste das seguintes etapas: (i) identificação do perigo (biológico, químico ou físico capaz de causar efeitos adversos à saúde e que possam estar presentes num alimento ou grupo de alimentos), (ii) caracterização do perigo (avaliação qualitativa e/ou quantitativa da natureza dos efeitos adversos à saúde associados com agentes biológicos, químicos e físicos que possam estar presentes em alimentos), (iii) avaliação da exposição, e (iv) caracterização do risco.

15 Dentre os 345 ingredientes ativos registrados para uso em diversas culturas, na Argentina, em 2007, apenas 110 (isto é, $31,8 \%$ ) possuíam LMRs estabelecidos pelo Codex (G/SPS/W/211).
} 
Além disso, os LMRs estabelecidos pelo Codex Alimentarius baseiamse em práticas agrícolas internacionais, que visam proteger e preservar a saúde humana, garantindo o comércio internacional de alimentos livres de barreiras ao parâmetro de resíduos. Entretanto, o nível de resíduos de defensivos em alimentos depende de fatores agronômicos e climáticos, e muitos países estabelecem seus próprios limites baseados nas boas práticas agrícolas praticadas localmente (IMOTO, 2004).

\section{Estudo de Caso: Limites Máximos de Resíduos para Trigo}

Tendo em vista a recomendação do CCPR, foi feito um estudo, com base nos limites máximos de resíduos (LMRs) praticados pelo Brasil, para a cultura de trigo, principal produto importado pelo Brasil, em 2008, no valor de US\$1.873.581.473.

Os LMRs de diversos ingredientes ativos, estabelecidos pelo Codex Alimentarius para o trigo, podem ser vistos na Tabela 1. Para efeitos analíticos, constam também na Tabela os LMRs desses ingredientes ativos ${ }^{16}$, praticados no Brasil.

${ }_{16}$ Além dos ingredientes ativos constantes da Tabela 5, o Codex Alimentarius estabelece LMRs (entre parênteses) para as seguintes substâncias, aplicadas na cultura do trigo: Aminopiralida $(0,10)$, Bioresmetrina $(1,00)$, Bitertanol (0,05), Ciprodinil (0,50), Clormequat (3,00), Clorpirifós Metílico (10,00), Etefon $(1,00)$, Famoxadona $(0,10)$, Fenbuconazol $(0,10)$, Imazalil $(0,01)$, Kresoxim Metílico $(0,05)$, Oxidemeton Metílico $(0,02)$, Quinoxyfen $(0,01)$ e Thiacloprid $(0,10)$. 
Tabela 1 - Limites Máximos de Resíduo dos ingredientes ativos, estabelecidos pelo Codex Alimentarius e pelo Brasil, para a cultura de trigo (elaboração própria, com base nos dados do MAPA, 2009, e do Codex, 2009).

\begin{tabular}{|c|c|c|}
\hline $\begin{array}{c}\text { INGREDIENTE ATIVI } \\
\text { DEFENSIVO }\end{array}$ & $\begin{array}{l}\text { Trigo - Codex } \\
\text { LMR (mg/Kg) }\end{array}$ & $\begin{array}{l}\text { Trigo - Brasil } \\
\text { LMR (mg/Kg) }\end{array}$ \\
\hline $2,4-\mathrm{D}$ & 2,00 & 0,02 \\
\hline Aldicarb & 0,02 & --- \\
\hline Bentazona & 0,10 & 0,01 \\
\hline Bifentrina & 0,50 & 0,60 \\
\hline Carbaril & 2,00 & --- \\
\hline Carbendazim & 0,05 & 0,10 \\
\hline Carbofuran & --- & 0,10 \\
\hline Carbosulfan & --- & 0,05 \\
\hline Clordane & 0,02 & --- \\
\hline Clorotalonil & 0,10 & 0,50 \\
\hline Clorpirifós & 0,50 & 0,20 \\
\hline Cipermetrina & 0,20 & --- \\
\hline Diazinon & --- & --- \\
\hline Difenoconazol & 0,02 & 0,05 \\
\hline Dimetenamida-P & --- & --- \\
\hline Dimetoato & 0,05 & 0,05 \\
\hline Diquat & 2,00 & --- \\
\hline Disulfoton & 0,20 & --- \\
\hline Fenpropimorfe & 0,50 & 0,30 \\
\hline Fipronil & 0,002 & 0,01 \\
\hline Glufosinato de amônio & --- & 0,05 \\
\hline Glifosato & --- & 0,05 \\
\hline Lindano & 0,01 & --- \\
\hline Malation & 0,50 & 8,00 \\
\hline Metidation & --- & --- \\
\hline Metiocarb & 0,05 & --- \\
\hline Metomil & 2,00 & 0,10 \\
\hline Metoxifenozida & --- & --- \\
\hline Paraquat & --- & 0,01 \\
\hline Forato & 0,05 & 0,05 \\
\hline Propargita & --- & --- \\
\hline Propiconazol & 0,02 & 0,10 \\
\hline Piraclostrobina & 0,20 & 0,50 \\
\hline Quintozene & 0,01 & --- \\
\hline Tebuconazol & 0,05 & 0,10 \\
\hline Terbufós & --- & --- \\
\hline Trifloxistrobina & 0,20 & 0,05 \\
\hline
\end{tabular}


Ao analisar a Tabela 1, é possível observar discrepâncias entre alguns ingredientes ativos dos defensivos permitidos para uso na cultura de trigo, pelo Codex Alimentarius, com aquelas permitidas pelo Brasil. Mesmo onde há concordância com o uso de determinado ingrediente ativo, constatam-se discordâncias com o valor do limite máximo de resíduo permitido para aquela substância.

Há 43,1\% de concordância entre as listas de ingredientes ativos autorizados pelo Codex e pelo Brasil; já com relação aos LMRs, há apenas 2 concordâncias (3,9\%), sendo que 27,3\% dos LMRs estabelecidos pelo Brasil são mais restritivos (valor inferior) do que os estabelecidos pelo Codex.

É importante ressaltar que, quanto menor o valor do LMR para dada substância, mais complexa é a sua determinação analítica, já que envolve, frequentemente, a necessidade do uso de materiais de referência certificados (MRC) ${ }^{17}$ (para calibração dos equipamentos) e de métodos de validação de ensaios.

No caso dos defensivos, o MRC ${ }^{18}$ é a mescla de uma matriz (que pode ser um hortifrutigranjeiro qualquer) com um ou mais tipos de defensivos agrícolas. Dado que o MRC já traz quais defensivos estão contidos nele e em que quantidades, o laboratório, necessariamente, precisará quantificar os resíduos destes em medidas mais próximas possíveis da determinada. Por exemplo: se a matriz do MRC for o tomate e sua concentração conhecida de um defensivo for de $0,1 \mathrm{mg} / \mathrm{Kg}$ da polpa daquele fruto, um ensaio nela realizado deverá indicar um número dentro de margens de erro aceitáveis, que esteja ao redor de $0,1 \mathrm{mg} / \mathrm{Kg}$.

\footnotetext{
${ }^{17}$ Material de Referência é o material ou substância que tem um ou mais valores de propriedades que são suficientemente homogêneos e bem estabelecidos para ser usado na calibração de um aparelho, na avaliação de um método de medição ou atribuição de valores a materiais. Um material de referência pode ser uma substância pura ou uma mistura, na forma de gás, líquido ou sólido.

18 Esquematicamente, pode-se dizer que os MRCs são formados por substâncias químicas integradas em uma matriz, que pode ser sólida, pastosa (Ex.: defensivos em frutas), líquida (Ex.: defensivos em água) ou gasosa (Ex.: dióxido de carbono em algum outro gás). A matriz do MRC deve ser mais próxima possível da matriz do material a ser submetido ao processo de medição, por exemplo, defensivos em solos.
} 
Em alguns casos, tem sido prática comum dos países desenvolvidos estabelecerem o valor do LMR coincidente como o menor nível em que os resíduos de defensivos sejam passíveis de detecção e quantificação num produto (conhecido como Limite de Quantificação, Limit of Determination - LOD, ou Zero Tolerance). Na maioria dos casos, quando um país estabelece o LOD como LMR de dado defensivo, isso reflete o fato de que não há usos aprovados da substância na cultura. Culturas que excedam esse nível não podem ser importadas.

Assim, a prática pode representar um obstáculo ao comércio para os países em desenvolvimento, que, em geral, não dispõem de infraestrutura laboratorial capacitada (em equipamentos e recursos humanos) para essas análises.

Um caso prático encontra-se registrado no site Global 21, sob o título "APREENSÃO - EUA apreendem lotes de Farinha Láctea Nestlé". Segundo a reportagem, um lote com 201 latas da mistura Farinha Láctea, produzido pela Nestlé, foi apreendido pelo Departamento de Proteção ao Consumidor do Estado de Connecticut, nos EUA, por conter níveis intoleráveis do defensivo pirimifos metílico. Como esse produto químico não é permitido em derivados do trigo nos EUA, não há padrões toleráveis à presença dele nos cereais (GALVÃO, 2009).

\section{Conclusões}

Muitos dos defensivos agrícolas que as nações industrializadas baniram ou restringiram, quer diretamente, quer indiretamente (em produtos e alimentos), ainda são usados largamente nos países em desenvolvimento. Assim, embora o Codex Alimentarius estabeleça padrões para os limites máximos de resíduos (LMR), outros países adotam suas próprias referências, o que pode gerar dificuldades na comercialização internacional de alimentos e tornar-se barreiras ao comércio. 
Como foi apresentado, a determinação dos limites máximos de resíduos em produtos, como o trigo, envolve o uso de um método analítico que tenha sido previamente validado. A validação de um método analítico deve ser realizada, sempre que for necessário verificar se o desempenho de seus parâmetros é adequado à utilização em determinada aplicação. A extensão requerida da validação ou da revalidação vai depender da natureza das alterações envolvidas. Métodos publicados ${ }^{19}$, mesmo por instituições internacionalmente reconhecidas, quando realizados pela primeira vez em um laboratório, precisam ser validados para verificar se os parâmetros estabelecidos pelo método são alcançados pelo laboratório.

A utilização de materiais de referência é parte crítica do processo de validação. Um certificado de análise, que caracterize completamente a substância de referência, deve acompanhar todo padrão analítico, seja ele material de referência certificado (MRC), seja um padrão de trabalho, sendo este último usualmente um padrão produzido no próprio laboratório, que é quantificado contra um material de referência certificado.

Contudo, materiais de referência, para uso em defensivos agrícolas, possuem alto custo de produção, já que as etapas de sua produção são demoradas e dispendiosas. Atualmente, não há materiais de referência certificados de alimentos produzidos no Brasil, o que pode ocasionar, por um lado, entraves comerciais por ocasião das exportações e, por outro, ingestão de produtos que contêm substâncias altamente tóxicas, por ocasião das importações.

O Brasil não dispõe de metodologia para quantificação de defensivos em cereais (trigo), e, mesmo para os produtos em que há metodologia disponível no país, há carência de recursos humanos de alta qualificação, imprescindíveis às análises.

${ }^{19}$ Para o setor de defensivos agrícolas, os requisitos de validação de métodos analíticos aplicáveis à determinação de teor de ingrediente ativo em produtos formulados e técnicos foram definidos no documento intitulado Guidelines on method validation to be performed in support of analytical methods for agrochemical formulations, emitido pela CIPAC (Collaborative International Analytical Pesticide Council). 
Assim, faz-se mister o desenvolvimento de materiais de referência e de metodologias que possibilitem a quantificação dos defensivos, especialmente em produtos nos quais o país tenha interesse estratégico. Além disso, é preciso investir na formação de recursos humanos capacitados para essas análises. Caso contrário, é possível antever um cenário onde o país enfrentará sérios obstáculos, no comércio internacional, às suas exportações de agroderivados.

\section{Referências}

Codex Alimentarius. Site Institucional. Disponível na internet em http:// www.codexalimentarius.net/web/index en.jsp. Arquivo acessado em 2009.

CX/PR 07/39/10. Discussion Paper about Enforcement of Codex MRLs (Prepared by The Netherlands). Codex Committee on Pesticide Residues. Thirty-ninth Session. Beijing, China, 7 - 12 May 2007.

FERREIRA, Celso P. Aspectos Toxicologicos e de Segurança no Manuseio de Agroquímicos. Série Técnica IPEF, Piracicaba, v.4, n.12, p.132 - 149, Set.1987.

FREITAS, Carlos Machado de. Avaliação de riscos como ferramenta para a vigilância ambiental em Saúde. Inf. Epidemiol. Sus, dez. 2002, vol.11, n'.4, p.227-239.

GALVÃO, Vinícius Queiroz. APREENSÃO - EUA apreendem lotes de Farinha Láctea Nestlé. Disponível na internet em http:// www.global21.com.br/materias/materia.asp?cod=22162\&tipo=noticia . Arquivo acessado em 2009.

G/SPS/W/211. Maximum Residue Levels for Pesticides - Impact on Exports from Developing Countries. Communication from Argentina. Committee on Sanitary and Phytosanitary Measures of WTO. 26 June 2007. 
IMOTO, Marvina Natsue. Validação de método multiresíduo para pesticidas organohalogenados em maçã por cromatografia gasosa com captura de elétrons (cg/ecd) e cromatografia gasosa com espectrometria de massa $(\mathbf{c g} / \mathbf{m s})$. Dissertação de Mestrado. Programa de Pós-Graduação em Tecnologia de Alimentos. Departamento de Engenharia Química. Universidade Federal do Paraná. Curitiba, 2004.

IPCS INCHEM. International Programme on Chemical Safety. Azocyclotin. Evaluations 1979. Pesticide Residues in Food - 1979. Joint meeting of the FAO Panel of Experts on Pesticide Residues in Food and the Environment and the WHO Expert Group on Pesticide Residues. Geneva, 3-12 December 1979. Disponível em http://www.inchem.org/ documents/jmpr/jmpmono/v079pr05.htm. Arquivo acesso em 2009.

MAPA. MINISTÉRIO DA AGRICULTURA, PECUÁRIA E ABASTECIMENTO. Secretaria de Relações Internacionais do Agronegócio. Balança Comercial do Agronegócio - 2008. Disponível na internet em http://www.agricultura.gov.br/pls/portal/url/ITEM/ 2CA7D34E3B3F53B9E040A8C075020715. Arquivo acessado em 2009.

NOVAES, Roberto J. S. Avaliação dos Riscos à Saúde dos Trabalhadores de uma Planta de Produção de Lubrificantes - Uma Proposta de Gerenciamento de Riscos. Dissertação de Mestrado. Escola Nacional de Saúde Pública. Fundação Osvaldo Cruz. Abril, 2000.

OLIVEIRA, Louise Antunes de. A Importância das Normas Internacionais para o Comércio da Fruticultura Brasileira. 2005. 168p. Dissertação (Mestrado em Ciências) - Escola Superior de Agricultura "Luiz de Queiroz". Universidade de São Paulo.

Post, Diahanna L. A Comissão do Codex Alimentarius e Normas de Inocuidade nos Alimentos. Universidade da Califórnia, Berkeley. Setembro, 2003. 


\begin{abstract}
Pesticides play an important role in agriculture, prevent loses by pests which destroy cultures and causes several damages to producers and to exporting countries of agriculture products. However, pesticides leave residues where they are used. A parameter used for residue quantification is known Maximum Residue Limit (MRL). This parameter is the most important, not only in terms of public health, but mainly international trade, causing many impacts that can represent, in some cases, barriers to trade between countries, especially when established values for a country differ from those established by international organizations. The present Article show how this parameter impacts agriculture products' trade of developing countries, based on case study.
\end{abstract}

Keywords: Pesticides, residue, MRL, international trade. 
REVISTA DE ECONOMIA E AGRONEGÓCIO, VOL.7, $N^{\circ} 2$ 